Original Paper http://ajol.info/index.php/ijbcs http://indexmedicus.afro.who.int

\title{
Imagerie échographique du développement embryonnaire chez la chèvre du Sahel
}

\author{
Boureima TRAORE, Moussa ZONGO*, T. Auguste YAMBOUE et \\ S. Drissa SANOU
}

Laboratoire de Physiologie animale, UFR/SVT, Université Ouaga I Pr Joseph KI-ZERBO, 03 BP 7021 Ouagadougou 03 - Burkina Faso.

*Auteur correspondant; E-mail : oussa_zongo59@yahoo.fr, moussa_zongo@univ-ouaga.bf ; Tél: (00226) 70259047

\section{RESUME}

Le contrôle de la gestation chez les chèvres constitue une voie importante pour organiser le rationnement des animaux, optimiser les productions animales et contribuer à l'atteinte de la sécurité alimentaire et nutritionnelle en Afrique subsaharien. La présente étude a pour objectif de déterminer les caractéristiques et la chronologie des séquences de développement embryonnaire et fotal chez les chèvres par échographie. Elle a concerné dix-huit $(n=18)$ chèvres du Sahel gestantes. Les examens ont été pratiqués deux fois par semaine au moyen d'une sonde transrectale de $5 \mathrm{Mhz}$ entre Jour $(\mathrm{J}) 18$ et 60 après les saillies. Les résultats obtenus montrent que, la vésicule embryonnaire de diamètre 9,42 $\pm 1,01 \mathrm{~mm}$ contenant un embryon filamenteux de longueur 7,37 $\pm 1,05 \mathrm{~mm}$ apparait en moyenne 23,80 $\pm 3,76$ jours avec des variations entre les primipares et les multipares. A J24, l'embryon se métamérise, s'épaissit et se transforme en bouton embryonnaire. Les battements cardiaques et le cordon ombilical ont été observés à J28. L'ossification de la voûte crânienne avec la délimitation des orbites ont été observés à J40. A partir de J45 de la gestation, les membres, la tête, la queue, le cordon ombilical, la colonne vertébrale et le tronc sont bien individualisés. A J48 les fœtus effectuent des mouvements de pattes. Les os des membres (tibia, fémur, humérus, cubitus) et vertèbres sont mesurables à $\mathrm{J} 54$ de la gestation. Ces données obtenues sont de précieux guides aux producteurs et aux techniciens dans le contrôle de la gestation des caprins.

(C) 2019 International Formulae Group. All rights reserved

Mots clés : échographie, embryon, développement, chèvre du Sahel.

\section{Ultrasonographic imaging of embryonic growth in Sahel goat}

\begin{abstract}
Pregnancy control in goats is an important way of organizing animal management, improving livestock productions and contributing to reach food and nutritional safety in sub-Saharan Africa. The present study aims to determine the characteristics and the chronology of the sequences of embryonic and foetal development in the goats by ultrasonography. It was carried out on eighteen $(n=18)$ pregnant Sahelian goats. Ultrasonographic exams were performed twice a week using a transrectal linear transducer of $5 \mathrm{MHz}$ beginning day 18 until day 60 of pregnancy. The obtained results show that in Sahelian goat, embryonic vesicle appeared on average 23.80 \pm 3.76 days with means diameter $9.42 \pm 1.01 \mathrm{~mm}$ and filamentous embryo whose length was $7.37 \pm 1.05 \mathrm{~mm}$.
\end{abstract}


In D24, the embryo grows, thickens and takes a spherical form called embryonic button. Heartbeats were detected and umbilical cord was observed firstly at day 28. The ossification of the skull-roof and the delimitation of the orbits were observed at day 40. At day 45, the limbs, head, tail, umbilical cord and body were very individualized. At day 48 the foetuses carry out movements of legs. Limbs bones of the members (tibia, femur, humerus, ulna) and vertebrae are measurable at day 54 of gestation..

These data are valuable guides with the producers and the technicians in pregnancy control of the gestation in goat.

(C) 2019 International Formulae Group. All rights reserved

Keywords: Ultrasound, Embryonic, development, Sahelian goat.

\section{INTRODUCTION}

Ces dernières années ont connu un renouveau des travaux sur les caprins. Dubeuf (2011) et Rege et al. (2011) soulignent l'intérêt pour la recherche de soutenir ce secteur d'activité agricole, notamment dans les régions où les perspectives de développement de l'élevage caprin semblent favorables. La chèvre du Sahel, grâce à de son cycle court et continue et son format, est mieux adaptée aux capacités d'investissement des familles modestes et constitue l'une des principales sources de protéines animales (viande, lait et ses dérivés) et de revenus pour les populations rurales (Zongo et al., 2009 ; Nantoumé et al., 2011).

Cette espèce développe cependant de nombreuses contraintes de reproduction dont la pseudogestation avec des moyennes comprises entre 1,3 et $10,5 \%$ (Barna et al., 2017 ; Almubarak et al., 2018). L'application de techniques de diagnostic précoce de gestation et de suivi du développement fœtal revêt une valeur ajoutée considérable pour optimiser le taux de survie des nouveaux nés et améliorer le revenu des producteurs (Karen et al., 2014). L'échographie permet de détecter les femelles gestantes, d'évaluer la viabilité embryonnaire et de compter le nombre de fœtus par gestation (Zongo et al., 2014 ; Samir et al., 2016).

Dans les élevages tropicaux où les mâles et les femelles sont conduits ensemble, cette technique est un atout important pour une gestion optimale de la reproduction. Elle fournit aux producteurs, les informations nécessaires pour regrouper les femelles gestantes selon leur besoin nutritionnel et organiser un rationnement approprié au cours du dernier trimestre de la gestation, réaliser le tarissement à des périodes adéquates et les préparer à la parturition (Vural et al., 2008).

L'application de l'échographie à la reproduction de la chèvre est encore récente et a porté sur la validation de la technique, le développement folliculaire et sur l'involution utérine au cours du postpartum (Zongo et al., 2014 ; Zongo et al., 2015). L'identification des femelles gestantes et le suivi du développement embryonnaire et fœtal n'ont pas suffisamment été développés.

L'objectif de la présente étude est de déterminer les caractéristiques et la chronologie des séquences de développement embryonnaire et fœtal chez la chèvre du Sahel par observation échographique chez dix-huit (18) femelles gestantes.

\section{MATERIEL ET METHODES Animaux et traitements}

L'étude a été réalisée à la station expérimentale de l'Université Ouaga I Professeur Joseph KI-ZERBO. Elle a concerné dix-huit $(n=18)$ chèvres d'âge, de poids et de parité respectifs compris dans les intervalles [24 - 36] mois, [30 - 40] kg et [14]. Toutes les femelles ont été induites en œstrus et saillies par cinq (05) boucs fertiles de la station expérimentale. Un diagnostic précoce de gestation par échographie a ensuite été réalisé à partir de dix-huit jours (J18) après la saillie. Le jour de la saillie est considéré comme le jour zéro (J0) de la gestation (Karen et al., 2014).

\section{Examens échographiques}

Les observations échographiques ont été pratiquées deux fois par semaine (Karen et 
al., 2014) du jour dix-huit (J18) au jour soixante (J60) après les saillies. Les femelles ont été maintenues en station débout dans un couloir de contention. Le diagnostic de gestation et les examens de l'embryon ont été réalisés par voie transrectale au moyen d'un échographe de marque CHISON 8500 muni d'une sonde linéaire de $5 \mathrm{MHz}$. Le câble de raccordement de la sonde a été rigidifié avec un manchon de tube en caoutchouc afin de faciliter son introduction et sa manipulation dans le rectum de l'animal. La sonde a été enduite d'un gel, avant tout examen. Les critères d'un diagnostic précoce de gestation sont ceux rapportés par Padilla-Rivas et al. (2005) et Karen et al. (2014).

Les délais moyens d'apparition des organes embryonnaire et fœtal notamment la tête, le tronc, les membres, les placentômes et les dates de l'édification des os ont été enregistrés.

Les dimensions des différents organes ont en outre été mesurées. Les images représentatives des séquences du développement embryonnaire et de la croissance fotale ont été enregistrées et commentées tout au long de l'expérimentation.

\section{Analyses statistiques}

Les résultats collectés ont été rangés en moyenne plus ou moins écart-type et analysés au moyen du test $t$ de student avec le logiciel GraphPad Prism 5.3. Les différences ont été considérées comme significatives au seuil de probabilité de 5\% $(\mathrm{p}<0,05)$. Les images échographiques ont été traitées à l'aide de Paint de MS Office 2016.

\section{RESULTATS}

\section{Diagnostic précoce de gestation}

Le délai moyen pour un diagnostic précoce de gestation chez la chèvre du Sahel est de 23,80 $\pm 3,76$ jours. Pour l'ensemble des femelles de l'expérimentation, les diagnostics précoces de gestation ont été réalisés dans l'intervalle [21 - 30] jours. Toutefois, $75 \%$ des diagnostics précoces de gestation ont été pratiqués à J24. Le délai moyen de diagnostic de gestation a varié selon la parité de la femelle. Chez les femelles primipares, le diagnostic de gestation semble plus précoce comparativement aux femelles multipares. Les délais moyens de diagnostic précoce de gestation ont été de 21,2 $\pm 1,83$ jours chez les primipares. Chez les femelles multipares, ce délai a été de 25,41 $\pm 2,75$ jours. La différence de variation entre les délais moyens de diagnostic précoce de gestation chez les primipares et multipares a été significative $(\mathrm{p}=0,04)$. Les observations échographiques de la gestation ont montré une augmentation du diamètre de la corne gestante et la présence de vésicules embryonnaires (Figure 1A). De J24 à J30, les diamètres moyens de la corne gestante sont passés de $27,53 \pm 2,76 \mathrm{~mm}$ à $33,73 \pm 5,20 \mathrm{~mm}$.

\section{Développement embryonnaire}

\section{Vésicule embryonnaire ou sac embryonnaire}

A partir du jour 21 jusqu'au jour 23 après les saillies, les observations échographiques ont rapporté l'apparition d'une vésicule remplie d'un liquide contenant un filament lumineux en son centre (Figure 1A). Ces observations ont été rapportées chez huit $(\mathrm{n}=8)$ femelles à $J 21$ et chez six $(\mathrm{n}=6)$ autres femelles aux jours 23 et 24 après les saillies. Le diamètre de la vésicule embryonnaire a été de 9,42 $\pm 1,01 \mathrm{~mm}$ et la longueur du filament lumineux a été 7,37 \pm $1,05 \mathrm{~mm}$ à $\mathrm{J} 21$.

\section{Bouton embryonnaire}

A Jour 24 (J24), le filament lumineux s'est étalé et épaissi à l'intérieur de la vésicule liquidienne (Figure 1B). De J24 à J29, les images échographiques ont montré un embryon arrondi à ovale avec dans la partie médiane une ligne de symétrisation et au pourtour une métamérisation apparente. Le cordon ombilical est apparu à $\mathbf{J} 28$ et a mesuré $2,91 \pm 0,94 \mathrm{~mm}$. J28 est également la date où les battements cardiaques sont perceptibles pour la première fois (Figure 1C).

A partir de $\mathrm{J} 30$, les observations ont montré une image d'un embryon complet chez toutes les femelles de l'expérimentation. La longueur moyenne de l'embryon à cette date est de 10,29 $\pm 1,53 \mathrm{~mm}$. A cette date, les observations échographiques ont montré 
l'image d'un embryon recourbé avec l'apparition de la membrane amniotique. Aussi, la tête et le cœur sont individualisés et visibles (Figure 1D).

A J36, la cavité abdominale de l'embryon a été visible sur les images échographiques, les ébauches des pattes ont été apparentes, le cordon ombilical et la queue se dessinent aisément (Figure 2A).

Les placentômes ont été observés à $\mathrm{J} 40$ sous forme de nodules ovales et éclaircies sur la paroi utérine (figure $2 \mathrm{~B}$ ). A cette date, le diamètre moyen des placentômes a été de 7,23 $\pm 1,1 \mathrm{~mm}$.

A J42, la face ventrale de l'embryon a été examinée. L'image a montré que l'abdomen, la tête, les membres antérieurs et postérieurs et la queue sont nettement visibles (Figure 2C). Les figures 1 et 2 rapportent les images échographiques du développement chronologique de l'embryon et de ses annexes.

\section{Séquence de l'ossification}

L'ossification a commencé par la tête et a été caractérisée par la détection de la plaque osseuse du crâne ou voute crânienne à J40. Cette image circulaire et bordée d'une couche fine blanche au départ évolue pour se scinder en deux lobes appelés pariétaux à partir de J55 (Tableau 1, Figures 2C, 3 et 4). Les boutons osseux des membres antérieurs et postérieurs non segmentés sont observés à l'échographie distinctement in vivo à partir de $\mathrm{J} 43$ (Tableau 1 et Figure 2, 3). Les parties ossifiées se présentent au stade précoce en des points blancs (hyperéchogènes) entourées de zones noires (anéchogènes). Les différents segments des membres (tibia, fémur, humérus et radius) ne sont mesurables qu'à partir de J50 et plus (Tableau 1 et Figure 2). Les os de la colonne vertébrale sont observables à partir de J45 en moyenne (Tableau 1 et Figure $3 \mathrm{~A})$. Les vertèbres sont individualisées à J50 (Tableau 1 et Figure 3B).

Tableau 1 : Séquence d'apparition et mesures échographiques de quelques structures osseuses fœtales.

\begin{tabular}{|c|c|c|}
\hline \multirow[t]{2}{*}{ Paramètres fotaux (mm) } & \multicolumn{2}{|c|}{ Première observation à l'échographie } \\
\hline & Taille (mm) & Age (jours) \\
\hline Tibia & $4,94 \pm 2,73[2,83-8,97]$ & $52,5 \pm 3,08[48-54]$ \\
\hline Fémur & $3,74 \pm 2,09[2,14-7,34]$ & $50 \pm 4,47[48-58]$ \\
\hline Humérus & $2,05 \pm 1,32[2-6,1]$ & $45,2 \pm 2,01[43-48]$ \\
\hline Os de la voute crânienne & $10,31 \pm 2,06[7,16-12,53]$ & $39,5 \pm 3,03[36-45]$ \\
\hline Membres Antérieurs & $2,17 \pm 1,01[1,7-3,20]$ & $42,3 \pm 3[39-45]$ \\
\hline Membres postérieurs & $4,03 \pm 1,32[3,02-5,35]$ & $40,02 \pm 4,1[36-45]$ \\
\hline Colonne vertébrale & $20,92 \pm 3,02[18,13-24]$ & $46,79 \pm 5,01[42-51]$ \\
\hline
\end{tabular}




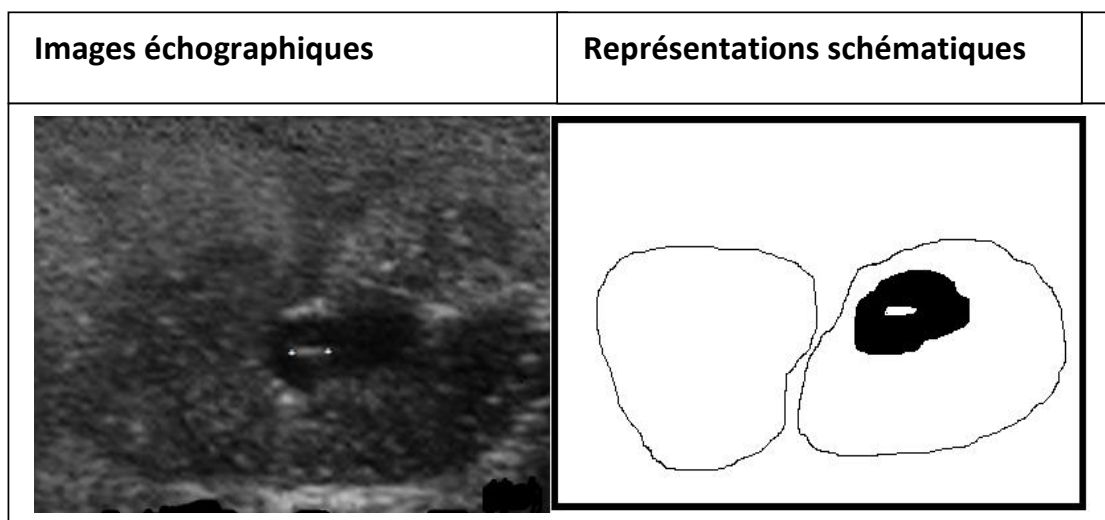

\section{Commentaires}

-Cornes utérines dissymétriques

-Vésicule sombre avec un embryon

filamenteux

1A: Jour 21 (J21)

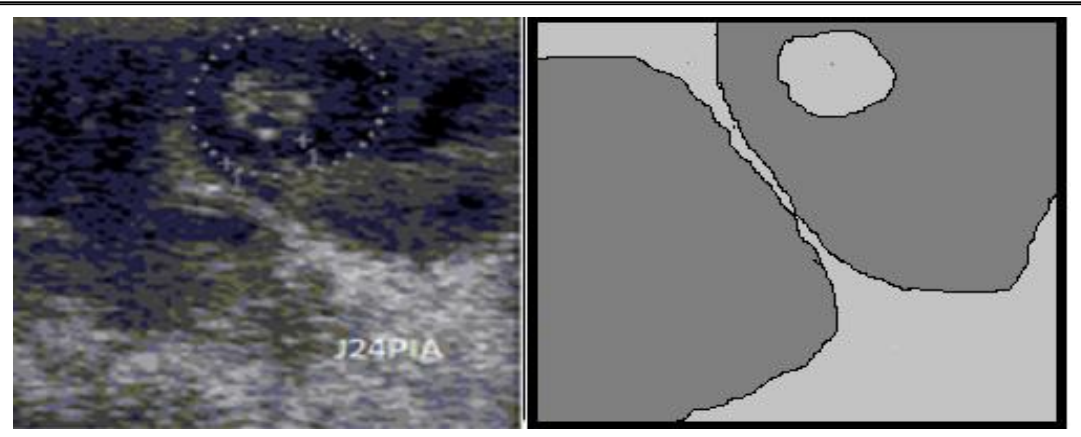

-Stade bouton embryonnaire

avec embryon segmenté

1 B : Jour 24 (J24)

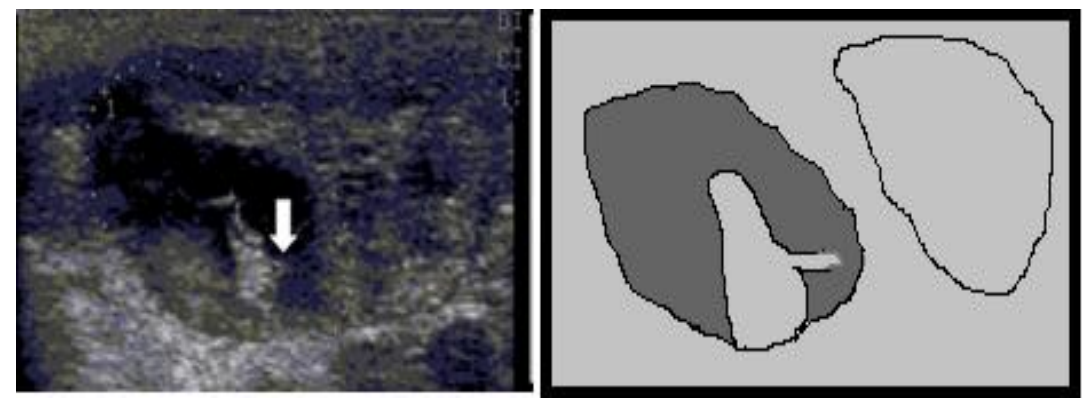

- Embryon

- Corne utérine vide

- Apparition du cordon

-Début battement cardiaque

1 C: Jour 28 (J28)
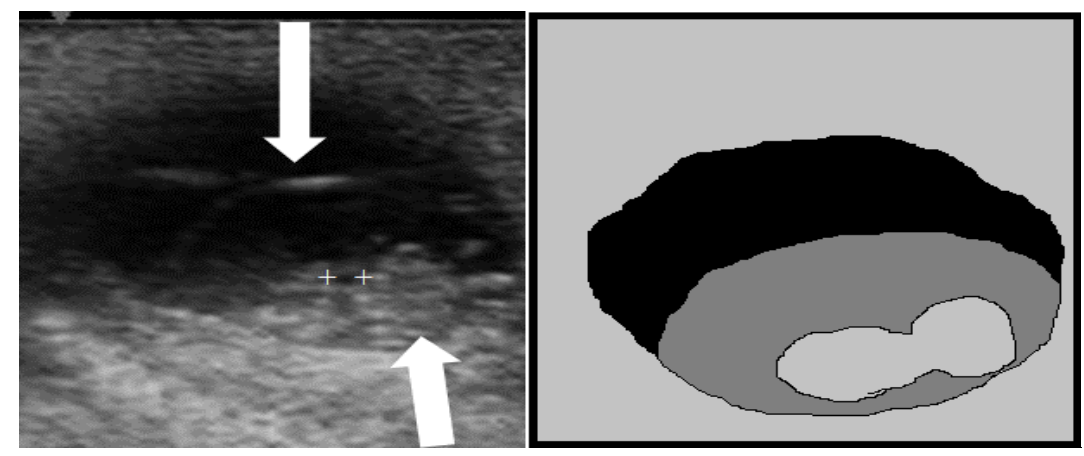

-Apparition membrane amniotique

-tête individualisée

-Cœur

1D: Jour 30 (J30)

Figure 1: Chronologie du développement embryonnaire au cours du premier mois. 


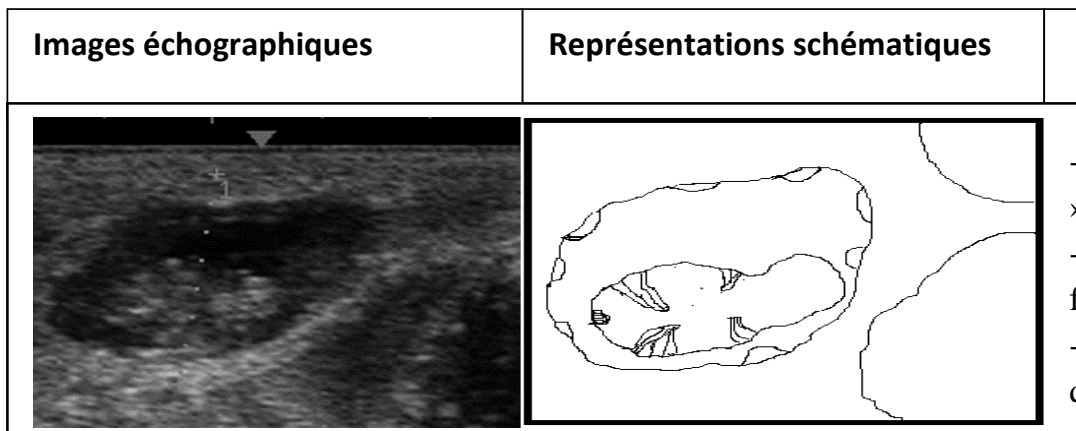

\section{Commentaires}

-Embryon en forme de « $\mathrm{L}$ » et libre,

-Cavité abdominale formée,

-Apparition des pattes, la queue et les cotylédons

2A : Jour 36 (J36)

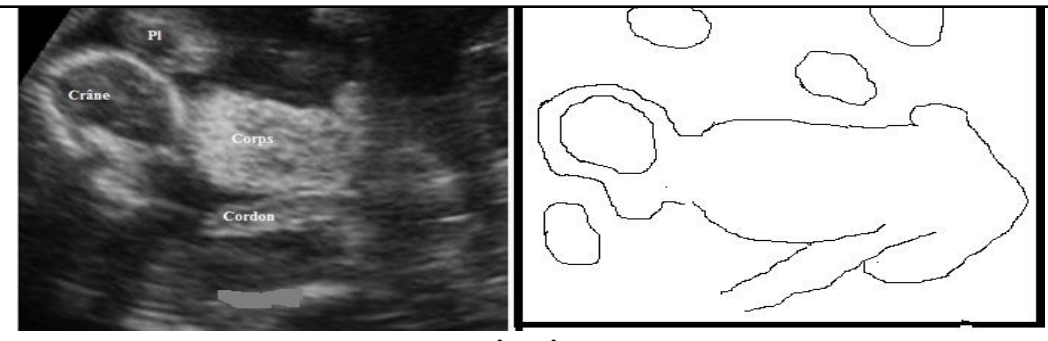

2 B : Jour 40 (J40)

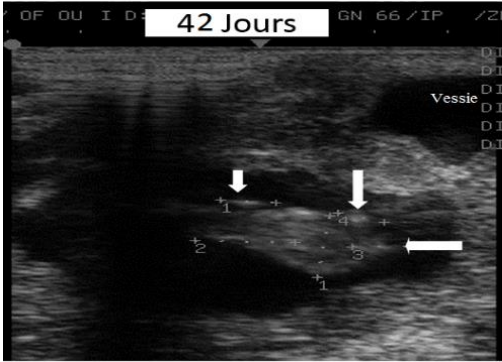

2C : Jour 42 (J42)
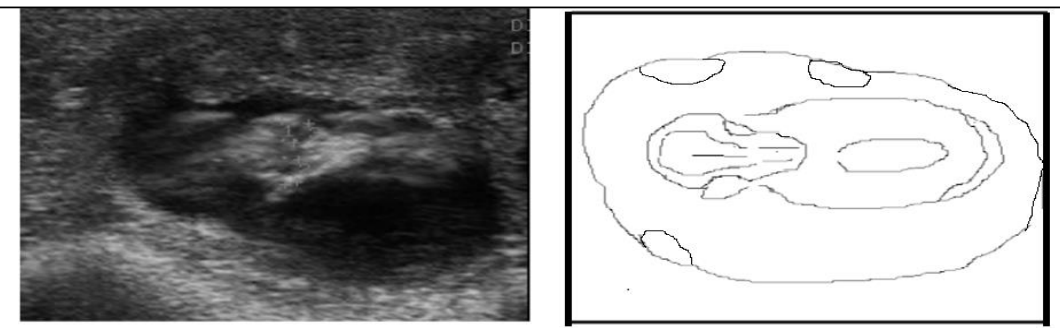

2D : Jour 45 (J45)
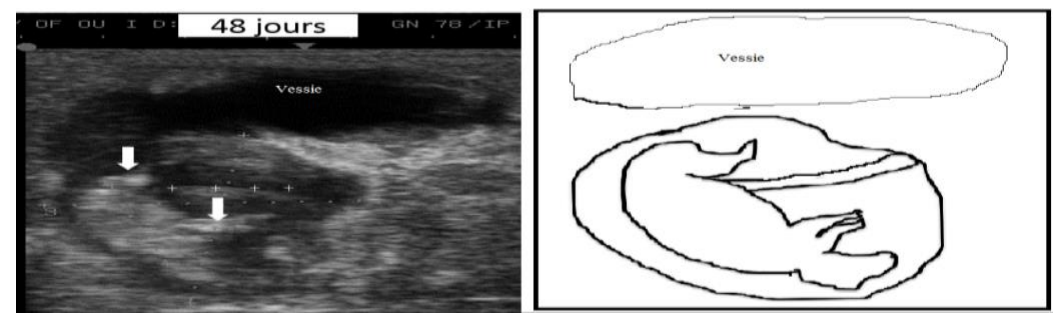

2 E : Jour 48 (48)
-Crâne délimité et le pariétal est visible

-Apparition des

placentômes

-Cordon bien

individualisé

Vue ventrale du fœtus de

42 jours

-Membres visibles

-Queue se dessine

- Cavité oculaire

bien délimitée

- Oreilles

- Grande mobilité

du fœtus

Vue de profile du fœtus de 48 jours

-Membres antérieurs et

postérieurs

-Cordon ombilical

- Tête

Figure 2 : chronologie du développement embryonnaire entre J36 et J48. 


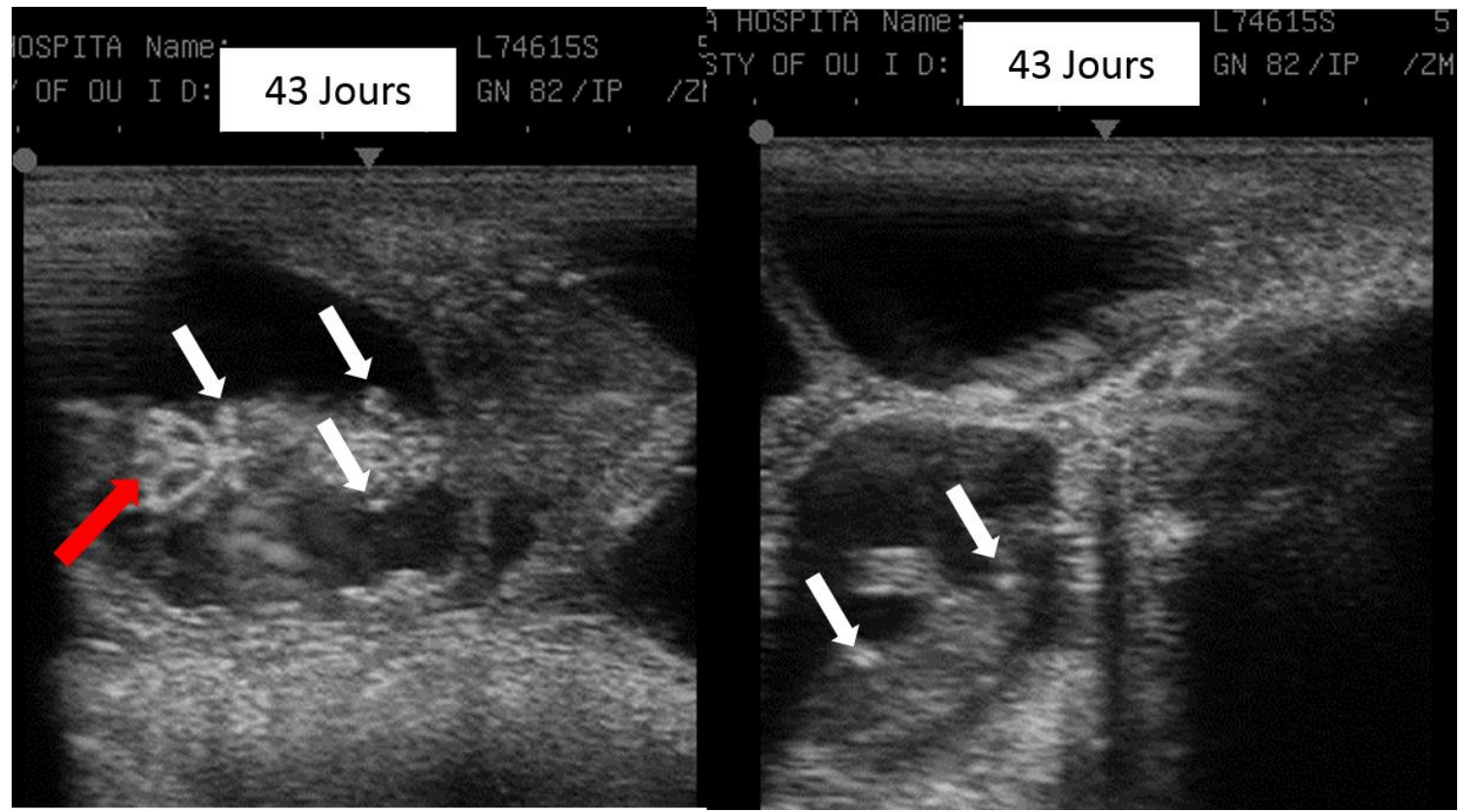

Figure 3 : Images échographiques mettant en évidence l'ossification du crâne et des membres d'un fœtus de 43 jours.
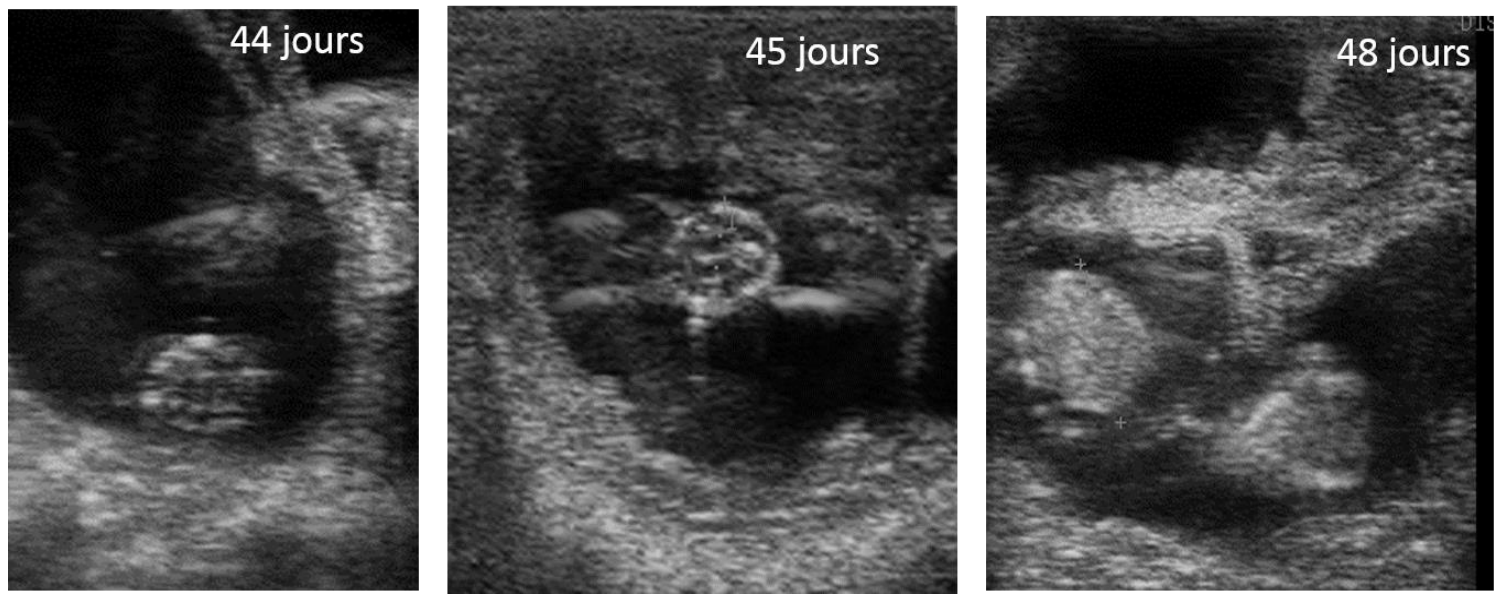

Figure 4 : Images échographiques du crâne à 44, 45 et 48 jours de gestation.

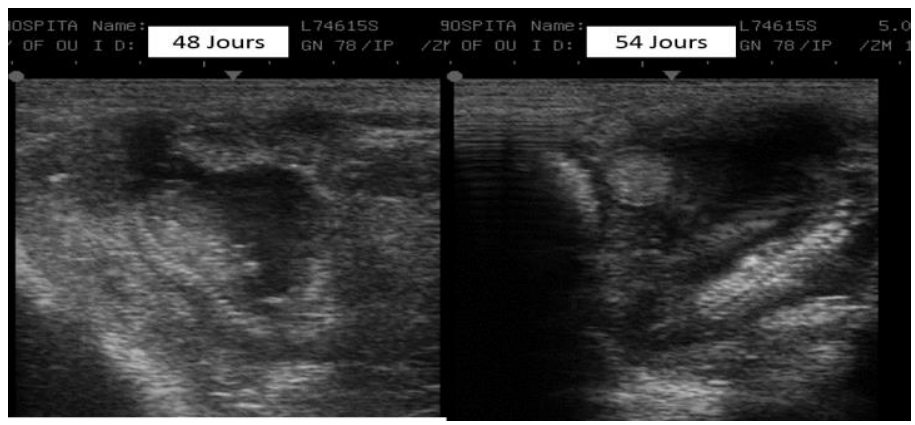

Figure 5 : Images échographiques de la colonne vertébrale à J48 et J54. 


\section{DISCUSSION}

Cette expérimentation a rapporté les résultats des examens échographiques de diagnostic précoce de gestation et du développement de l'embryon chez la chèvre du Sahel au cours des 60 premiers jours de gestation. Les représentations schématiques et l'analyse des observations échographiques ont permis de décrire les caractéristiques d'un développement embryonnaire précoce et tardif. Ces résultats sont complémentaires des données rapportées par Waziri et al. (2012) sur les observations post-mortem des fœtus de chèvres.

La fenêtre optimale pour le diagnostic précoce de gestation chez les femelles de notre expérimentation a été l'intervalle [21 30] jours. Cet intervalle correspond aux fourchettes de [24-29] et de [21 - 50] jours rapportées respectivement par Karen et al. (2014) et par Raja-Khalif et al. (2014) sur les chèvres Baladi et Boer.

Les observations échographiques ont montré que le début de gestation chez la chèvre du Sahel a été caractérisé par une augmentation du diamètre de la corne gestante et la présence d'une vésicule sombre contenant un embryon filamenteux. L'aspect filamenteux de l'embryon résulterait du processus d'élongation du blastocyste juste avant son implantation (Suguna et al., 2008). Le délai d'apparition de cette observation a varié en fonction de la parité des femelles. Elle a été observée précocement chez les femelles primipares et tardivement chez les femelles multipares $(\mathrm{P}<0,05)$. Cette différence serait liée à une modification anatomomorphologique de l'appareil génital sous la pression des gestations antérieures qui plonge l'utérus dans la cavité abdominale. De même, certains auteurs ont rapporté que la sensibilité de la technique échographique appliquée au diagnostic de gestation était plus élevée chez les jeunes que les vieilles brebis (Karen et al., 2004) et les chèvres (Karen et al., 2014). Ce facteur de variation disparaît avec la mise à jeun des animaux ou le soulèvement de l'abdomen (Karen et al., 2014).

Les caractéristiques de l'embryon observées à J24 chez la chèvre du Sahel ont été rapportées par Medan et al. (2004). Cette forme discontinue de l'embryon traduirait une métamérisation et une symétrisation apparente assimilable à l'organisation du corps, aux ébauches des membres et de la tête. Dans la présente étude, les battements cardiaques ont été observés à J28. La littérature sur les races caprines d'Europe et d'Asie a rapporté l'observation des battements cardiaques entre les jours 19 et 28 de gestation (Medan et al., 2004, Padilla-Rivas et al., 2005 ; Suguna et al., 2008). Bien que les battements du cœur soient perceptibles, les différentes cavités du cœur ne sont pas nettement observables. Suguna et al. (2008) ont rapporté des observations similaires. Le cordon ombilical a été observé à J28 avec un diamètre moyen de 2,91 $\pm 0,94 \mathrm{~mm}$. Des observations similaires ont été rapportées par Kandiel et al. (2015) chez les chèvres Shiba. Le cordon ombilical a une structure anatomique particulière qui le rend facilement observable à l'échographie. A J30, l'embryon est détaché de la paroi de l'utérus et la membrane amniotique est bien individualisée. Des observations similaires ont été rapportées par Raja-Khalif et al. (2011) chez la chèvre. Par ailleurs, l'embryon présente une forme recourbée qui pourrait s'expliquer par l'individualisation de la tête. La tête individualisée et différenciée du reste du corps par un étranglement a été observée à partir de J30. Chez les brebis, Valasi et al. (2016) ont rapporté que la tête et le tronc s'individualisent vers le J31.

Les placentômes ont été observées dans l'intervalle [36 - 42] jours de gestation avec un diamètre moyen de 7,23 $\pm 1,1 \mathrm{~mm}$. Ces observations ont été inclues dans la fourchette de 34 à 42 jours de gestation déjà rapportée chez la chèvre (Kandiel et al., 
2015 ; Medan et al., 2004 ; Suguna et al., 2008) et la brebis (Jones et al., 2016 ; Ali et Hayder, 2007). Les caractéristiques morphologiques des placentômes décrites par échographie sont celles des observations visuelles directes après l'abattage (Igwebuike et Ezeasor, 2013). Les placentômes résultent de l'interdigitation entre les cotylédons avec les caroncules utérines afin de faciliter les échanges foeto-maternelles (Igwebuike et Ezeasor 2013).

Le suivi par échographie de la séquence de l'ossification du fotus de chèvre est très important pour détecter les anomalies de développement et mieux comprendre la reproduction d'une espèce animale. Jadis étudié par radiographie, la technique échographique est devenue la méthode fiable et non invasive pour détecter et suivre la séquence de l'ossification de l'embryon et du fotus chez les ovins (Ali et Hayder, 2007). Dans cette étude, le crâne avec la délimitation des orbites a été observé pour la première fois en moyenne à J39. Cette observation laisserait penser que l'ossification de l'embryon a commencé par la formation de la voûte crânienne ou calvarium à partir du jour 42 (Waziri et al., 2012). Cette date est tardive par rapport à celles trouvées (36 jours) Valasi et al. (2016) et plus précoces à celles rapportées chez les ovins de races Ossimi (44) (Ali and Hayder, 2007) mais voisine de celles rapportées directement chez la chèvre du Sahel après abattage par (Waziri et al., 2012). Ces auteurs ont trouvé que le calvarium est membraneux et transparent à la $6^{\text {ème }}$ semaine d'âge. Cet os de forme circulaire au départ, évolue rapidement pour prendre une forme ovale bilobée et hyperéchogène vers le $55^{\text {ème }}$ jour de gestation comme déjà rapporté par Valasi et al. (2016) par échographie chez les moutons et après abattage chez la chèvre du Sahel (Waziri et al., 2012).

Les centres d'ossification des membres ont été mis en évidence en moyenne à partir du $40^{\text {ème }}$ et le $42^{\text {ème }}$ jour respectivement pour les membres postérieurs et antérieurs. Ces résultats sont légèrement tardifs comparativement à ceux rapportés (36 ${ }^{\text {ème }}$ jour) chez les ovins par Valasi et al. (2016). Cette différence pourrait s'expliquer par l'effet espèce et l'effet opérateur. Les os longs tels que l'humérus, le fémur et le tibia ont été observés respectivement à 48,50 et 52 jours d'âge. De plus, à cette période des mouvements de faibles amplitudes ont été observés. Des observations similaires ont été rapportées chez les ovins (Ali et Hayder, 2007; Jones et al., 2016 ; Valasi et al., 2016).

Ces observations correspondent à celles (49 jours) déjà rapportées par radiographie sur la séquence chronologique d'apparition des os pelviens et thoraciques chez la chèvre en Inde (Parmar et al., 2009 ${ }^{\mathrm{a}}$; Parmar et al., 2009 ${ }^{\mathrm{b}}$ ). Leurs tailles correspondent aux observations déjà rapportées chez d'autres races caprines (Mahdi et Khojasteh, 2012). Cependant, de légères différences existent entre la technique radiographique et celle échographique sur la détection, la netteté et la précocité dans le suivi de l'ossification. En effet, les petits os des membres antérieurs et postérieurs n'ont pas été identifiés nettement par échographie dans la présente étude tandis qu'ils ont été bien individualisés par radiographie (Parmar et al., 2009 ${ }^{\mathrm{a}}$; Parmar et al., 2009 ${ }^{\mathrm{b}}$ ). Aussi, la méthode échographique détecterait plus tôt les centres d'ossification que la radiographie comme déjà observé chez la chèvre (Parmar et al., 2009 ${ }^{\mathrm{a}}$ ). En somme, nos observations ont été plus tardives que celles rapportées chez les ovins et les caprins (Youssef et al., 2016).

Les différentes observations échographiques de la présente étude complètent les observations post-mortem décrites chez les chèvres (Waziri et al., 2012). La chronologie du développement des organes embryonnaires dans l'intervalle de nos observations correspond aux résultats 
rapportés par Waziri et al. (2012) et Valasi et al. (2016).

\section{Conclusion}

Ce travail a rapporté les images échographiques d'un diagnostic précoce de gestation et du développement embryonnaire chez la chèvre du Sahel. L'analyse des images a permis de décrire les caractéristiques d'une gestation précoce, du développement embryonnaire et fœtal chez la chèvre. Les données obtenues dans cette étude, constituent des valeurs de référence pour le contrôle du développement intra-utérin de plusieurs paramètres de l'embryon et du fœetus de chèvre. Elles servent, en outre, de précieux guides aux producteurs et aux techniciens dans la conduite des troupeaux caprins notamment pour le diagnostic de gestation, le suivi de la croissance fœtale, l'estimation de la date de parturition, la détection des pseudogestations et la détection des retards de croissance fœtale.

\section{CONFLIT D'INTERETS}

Les auteurs déclarent qu'il n'y a aucun conflit d'intérêts.

\section{CONTRIBUTIONS DES AUTEURS}

BT est l'investigateur principal des travaux, il a rédigé l'article, analysé et interprété les données. MZ a défini le sujet et encadré le protocole scientifique, Il a corrigé l'article et a soumis à publication. AY a participé à la collecte et analyse des données, il a ensuite revue la bibliographie. DS a participé à la définition du sujet et est le coordonnateur de l'unité de recherche.

\section{REMERCIEMENTS}

Ce travail a été réalisée grâce à l'appui technique et financier de l'Agence International pour l'Energie Atomique (AIEA, BKF 5008) / country program for Support development of small ruminants production in
Burkina Faso. Les auteurs voudraient exprimer toute leur gratitude à cette institution pour leur accompagnement. Ils expriment leurs sincères remerciements et reconnaissance au Professeur Christian Hanzen de l'Université de Liège pour ses conseils avisés et sa bienveillante attention. Nous remercions le Professeur WERE PITALA pour sa participation à la définition du sujet et à la relecture du manuscrit.

\section{REFERENCES}

Ali A, Hayder M. 2007. Ultrasonographic assessment of embryonic, fetal and placental development in Ossimi sheep. Journal Small Ruminant Research, 73: 277-282.

DOI:

10.1016/j.smallrumres.2007.01.011

Almubarak AM, Abass NA, Badawi ME, Ibrahim MT, Elfadil AA, Abdelghafar RM. 2018. Pseudopregnancy in goats: Sonographic prevalence and associated risk factors in Khartoum State, Sudan. Veterinary World, 11(4): 525-529. DOI: 10.14202/vetworld.2018.525-529

Barna T, Apić J, Bugarski D, Maksimović N, Mašić A, Novaković Z, Milovanović A, 2017. Incidence of hydrometra in goats and therapeutic effects. Arhiv Veterinarske Medicine, 10 (1): 13 - 24.

Dubeuf JP. 2011. The social and environmental challenges faced by goat and small livestock local activities: Present contribution of researchdevelopment and stakes for the future. Small Rum. Res., 98: 3-8. DOI: 10.1016/j.smallrumres.2011.03.008

Igwebuike UM, Ezeasor DN. 2013. The morphology of placentomes and formation of chorionic villous trees in West African Dwarf goats (Capra hircus). Veterinarski Arhiv., 83(3): 313-21. DOI:10.5958/2277940X.2015.00043.1 
Jones AK, Gately RE, McFadden KK, Zinn SA, Govoni KE, Reed SA. 2016. Transabdominal ultrasound for detection of pregnancy, fetal and placental landmarks, and fetal age before Day 45 of gestation in the sheep. Theriogenology, 85: 939-945.

Kandiel MMM, Watanabe G, Taya K. 2015. Ultra sonographic assessment of fetal growth in miniature "Shiba" goats (Capra hircus). Animal Reproduction Science, 162: 1-10.

Karen A, Samir H, Ashmawy T, El-Sayed M. 2014. Accuracy of B-mode ultrasonography for diagnosing pregnancy and determination of fetal numbers in different breeds of goats. Animal Reproduction Science, 147: 2531.

DOI:

10.1016/j.anireprosci.2014.03.014

Karen A, Szabados K, Reiczigel J, Beckers J

F, Szenci O. 2004. Accuracy of transrectal ultrasonography for determination of pregnancy in sheep: effect of fasting and handling of the animals. Theriogenology, 61: 1291-1298. DOI:

10.1016/j.theriogenology.2003.07.018

Mahdi S, Khojasteh B. 2012. Prenatal development of Iranian goat fetuses, 3(10): 2022-2024. DOI: http://www.irjabs.com/files_site/paperlis t/r_152_121009134520

Medan M, Watanabe G, Absy G, Sasaki K, Sharawy S, Taya K. 2004. Early pregnancy diagnosis by means of ultrasonography as a method of improving reproductive efficiency in goats. Journal of Reproduction and Development, 50: 391-397.

Nantoumé H, Kouriba A, Diarra C, Coulibaly D. 2011. Amélioration de la productivité des petits ruminants : Moyen de diversification des revenus et de lutte contre l'insécurité alimentaire. Livestock Research for Rural Development, 23 (5).

Padilla-Rivas GR, Sohnrey B, Holtz W. 2005. Early pregnancy detection by real-time ultrasonography in boer goats. Small Ruminant Research, 58: 87-92. DOI: doi.org/10.1016/j.smallrumres.2004.09.0 04

Parmar VK, Patel KB, Desai MC, Mistry JN, Chaudhary SS. 2009 ${ }^{\mathrm{a}}$. Radiographic study on first appearance of ossification centers of bones in the goat fetuses: the thoracic limb. Indian Journal of Field Veterinarians, 4(3) : 53-56.

Parmar VK, Patel KB, Desai MC, Mistry JN, Chaudhary SS. 2009 $9^{\mathrm{b}}$. Radiographic study on first appearance of ossification centers of bones in the goat fetuses: the pelvic limb . Indian Journal of Field Veterinarians, 4(4) : 6-10.

Raja-Khalif RIA, Mohd-Nizam AR, Abdullah RB, Wan Khadijah WE. 2011. Using fetal-heart size measured from ultrasound scanner images to estimate age of gestation in goat. J. Anim. Vet. $A d v ., \quad$ 10: $2528-2540 . \quad$ DOI: 10.3923/javaa.2011.2528.2540

Raja-Khalif RIA, Rahman MM, WanKhadijah WE, Abdullah RB. 2014. Pregnancy diagnosis in goats by using two different ultrasound probes. Journal of Animal and Plant Sciences, 24(4): 1026-1031.

Rege JEO, Marshall K, Notenbaert A, Ojango JMK, Okeyo AM. 2011. Pro-poor animal improvement and breeding - what can science do? Livestock Science, 136: 1528.

Samir H, Karen A, Ashmawy T, Abo-Ahmed M, El-Sayed M, Watanabe G. 2016. Monitoring of embryonic and fetal losses in different breeds of goats using realtime B-mode ultrasonography. Theriogenology. 85(2): 207-215. DOI: 10.1016/j.theriogenology.2015.09.039 
Suguna K, Mehrotra S, Agarwal SK, Hoque M, Singh SK, Shanker U, Sarath T. 2008. Early pregnancy diagnosis and embryonic and fetal development using real time B mode ultrasound in goats. Small Ruminant Research, 80: 80-86. DOI:

https://doi.org/10.1016/j.smallrumres.20 08.10 .002

Valasi I, Barbagianni MS, Ioannidi KS, Vasileiou NGC, Fthenakis GC, Pourlis A. 2016. Developmental anatomy of sheep embryos, as assessed by means of ultrasonographic evaluation. Small Ruminant Research, 152: 56-73. DOI:10.1016/j.smallrumres.2016.12.016.

Vural MR, Sel T, Karagul H, Ozenc E, Orman M, Izgur H, Kuplulu S. 2008. Ultrasonographic examinations of embryonic fetal growth in pregnant Akkaraman ewes fed selenium supply and dietary selenium restriction. Revue Médecine Vétérinaire, 159(12): 628-633. Waziri MA, Sivachelvan NM, Mustapha AR, Ribadu AY. 2012. Time-related and sequential developmental horizons of Sahel goat fetuses. Sokoto Journal of Veterinary Sciences, 10(2): 32-39.
Youssef GBHB, El-aliem NMA, El-shewy EA. 2016. The relation between the growth plate closure in tibia and the age of sheep and goat: Medicolegal study'. Scholars Journal of Applied Medical Sciences, 4(3C): 812-815.

Zongo M, Traoré B, Ababneh MM, Hanzen C, Sawadogo L. 2015. Ultrasonographic assessment of uterine involution and ovarian activity in West Africa Sahelian goats. Journal of veterinary Medicine and Animal Health, 7(2): 71-76. DOI: https://doi.org/10.5897/JVMAH2014. 0322.

Zongo $\mathrm{M}$, Teresa $\mathrm{M}$, Rubio $\mathrm{P}$, Boly $\mathrm{H}$, Ababneh MM, Sanou D, Belemtougri R, Sawadogo L. 2014 : Évaluation de la technique de sexage et de fœtométrie par échographie chez la chèvre du Sahel. Int. J. Biol. Chem. Sci., 8(6): 2516-2522. DOI :

http://dx.doi.org/10.4314/ijbcs.v8i6.14 Zongo M, Pellecer-Rubio M, Meyer C. 2009. Caractérisation des élevages ovins et caprins en zone soudano-sahélienne du Burkina Faso. Annales Université Ouagadougou, Série C, 7: 93-130. 